\title{
Probiotics (Lactobacillus acidophilus and Bifidobacterium bifidum) prevent NEC in VLBW infants fed breast milk but not formula
}

\author{
Andreas Repa', Margarita Thanhaeuser', David Endress², Michael Weber ${ }^{3}$, Alexandra Kreissl', Christoph Binder' , \\ Angelika Berger ${ }^{1}$ and Nadja Haiden ${ }^{1}$
}

BACKGROUND: Specific probiotics prevent necrotizing enterocolitis (NEC). A mixture of lactobacilli and bifidobacteria (Infloran) was highly effective in Asian very-low-birth-weight (VLBW) infants. We analyzed the effect of Infloran on NEC, NEC severity, and the influence of enteral feedings (breast milk vs. formula) on NEC prevention in a cohort of European VLBW infants. METHODS: Infloran was implemented for routine use at our department. VLBW infants receiving probiotics were prospectively followed (2010-2012) and compared with historic controls (2008-2009). Data on NEC, neonatal morbidity, feeding tolerance, and descriptive parameters on NEC cases were analyzed.

RESULTS: Infloran had no statistically significant impact on NEC (controls: 24/233 (10.3\%); probiotics: 16/230 (7\%); $P=0.2$ ). However, NEC was significantly reduced in infants of the probiotics group who were fed any breast milk (20/179 (11.2\%) vs. $10 / 183(5.5 \%) ; P=0.027)$, whereas it was ineffective in infants exclusively fed formula (4/54 (7.4\%) vs. $6 / 44$ (13.6\%); $P=0.345)$. Occurrence of severe NEC (IIIb), time until full feeds, and gastric residuals were similar.

CONCLUSION: Infloran was of lower efficacy in a European VLBW cohort and showed a reduction of NEC only in infants fed breast milk. Future studies should investigate the influence of feeding formula or breast milk on the effect of probiotics.

N ecrotizing enterocolitis (NEC) is an important cause of mortality and morbidity in premature infants (1). Although its pathogenesis is incompletely understood, a relation to an immature intestinal barrier, aberrant bacterial colonization, and enteral feedings is well established (2). Due to the seriousness of the disease, preventive strategies like the use of human milk and standardized feeding protocols (3-5) are of highest interest.

Probiotics are microorganisms that confer a health benefit after ingestion (6). They are generally regarded as safe, and only single cases of sepsis were described in patients with major comorbidities (7). In preterm infants, probiotics may act beneficially by improving the intestinal barrier (8) and promoting a healthy gut microflora (9). Furthermore, they exert antiinflammatory effects on the gut epithelium (10) and improve local and systemic immune responses (11) that may strengthen a preterm infant's still immature immune defense. In the recent past, a preventive effect against NEC was assumed (12), and a variety of probiotic preparations were studied in randomized trials. Meta-analyses of these trials reported on a reduction of NEC in very-low-birth-weight (VLBW) infants by $65 \%(13,14)$, and the use of probiotics in clinical routine was recommended. Based on these suggestions, we decided to introduce probiotics for NEC prophylaxis as standard of care at our department. The probiotic preparation Infloran (Laboratorio Farmaceutico, Mede, Italia) consists of a mixture of Lactobacillus acidophilus and Bifidobacterium infantis and was chosen as its use was suggested by European experts (14) and it was available in sufficient quantities for routine application. Infloran (Laboratorio Farmaceutico) was highly effective ( $-80 \%$ NEC incidence) in a prospective randomized trial performed by Lin et al. (15) in China. Despite the high efficacy in this trial (14), we felt that it was important to evaluate the effect in our mainly European population of VLBW infants, as there are no published data on its use outside Asia. Furthermore, there is a lack of data on possible interactions of feeding breast milk vs. formula on the protective effect of probiotics. We therefore performed a prospective observational study and aimed at investigating the effect of Infloran (Laboratorio Farmaceutico) on the NEC incidence in VLBW infants after implementation at our unit. Secondary aims were to assess the influence of Infloran on NEC severity and whether the type of enteral feeding (breast milk vs. formula) modulates the effect of Infloran on NEC.

\section{RESULTS}

Screening

A total of 666 VLBW infants, <34/0 wk gestation, who were born between January 2008-December 2009 and October 
2010-July 2012 were screened for eligibility: 203 infants (control group: $n=107$; probiotics group: $n=96$ ) were excluded (for detailed reasons for exclusion, see Figure 1) and 463 infants (control group: $n=233$; probiotics group: $n=230$ ) were analyzed (Figure 1).

\section{Demographic and Basic Clinical Parameters}

Infants in the probiotics group were born with a significantly lower gestational age (GA) $(-10 \mathrm{~d} ; P=0.004)$ and birth weight $(-80 \mathrm{~g} ; P=0.020)$ than controls (Table 1). Their 5-min Apgar scores were lower ( 8 vs. $9 ; P<0.001$ ), but umbilical $\mathrm{pH}$ was higher (7.32 vs. $7.28 ; P<0.001)$. They received a higher amount of total fluids in the first week of life $(+5.8 \mathrm{ml} ; P=0.005)$ and were less often provided with umbilical catheters. Surfactant use was significantly higher $(73.9$ vs. $57.1 \% ; P<0.001)$ in the probiotics group. All other demographic (small for gestational age, prenatal steroids, antibiotics, preeclampsia/hypertonia, and cesarean section) and basic clinical parameters (closure and treatment of patent ductus arteriosus and use of vasopressors $<72 \mathrm{~h}$ after birth) were similar between the groups.

\section{Neonatal Outcome Parameters}

The primary outcome NEC in the probiotics group (7.0\%) was $-32 \%$ lower than that in the control group $(10.3 \%)$, but the result was not statistically significant $(P=0.200)$-also after correction for confounding variables (birth weight, any mothers' milk in the first 2 wk of life, premature rupture of membranes, prenatal antibiotics, and death) in a model of binary logistic regression (Tables 2 and 3). Mortality was significantly lower in the probiotics group ( 7.0 vs. $12.9 \% ; P=0.036)$; the compound outcome of "intraventricular hemorrhage (IVH) grade III/IV and/or NEC" was significantly lower (28/230 (12.2\%) vs. $47 / 232(20.3 \%) ; P=0.023)$. Retinopathy of prematurity requiring treatment was significantly higher in the univariate analysis (16.1 vs. $9.0 \% ; P=0.009)$ but not after correction for relevant confounders (GA and mortality; $P=0.401$; complete model not shown). Further outcome variables were not statistically different. No case of sepsis with a probiotic germ occurred. There was no statistically significant effect of

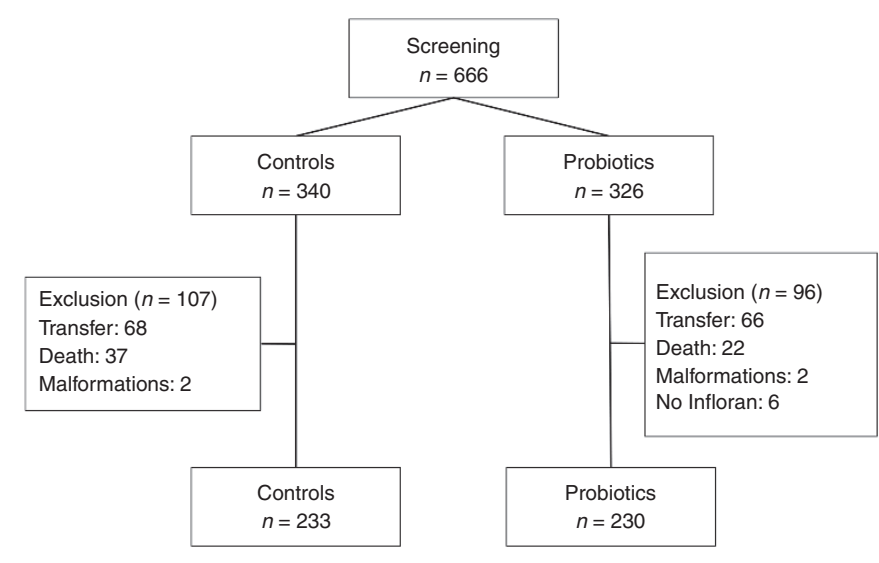

Figure 1. Flow chart showing the screening of very-low-birth-weight infants for study eligibility with reasons for study exclusion. probiotics in small-for-gestational-age infants on NEC incidence $(5 / 53(9.4 \%)$ vs. $4 / 38(10.5 \%) ; P=0.566)$.

\section{NEC Severity}

Administration of Infloran (Laboratorio Farmaceutico) had no impact on mortality of infants who developed NEC or occurrence of perforated NEC (IIIb) (Table 4). Laboratory parameters did not differ except for significantly lower platelets in the probiotics group. Clinical and radiological parameters did not vary.

\section{Nutritional Variables}

There was no difference in type and volume of enteral feedings between the two groups (Table 5). Moreover, intake of Infloran (Laboratorio Farmaceutico) had no influence on feeding tolerance (time until full enteral feeds, amount of gastric residuals, and presence of bilious gastric residuals).

\section{Subgroup Analysis According to Feeding Type}

A subgroup analysis of the effect of Infloran (Laboratorio Farmaceutico) according to the enteral feeding type (any breast milk vs. exclusively formula) during the first 2 wk of life showed a statistically significant impact on the development of NEC: Breast milk and Infloran (Laboratorio Farmaceutico) were protective $(P=0.027)$, whereas formula and Infloran (Laboratorio Farmaceutico) were ineffective (Tables 6 and 7). A quantitative analysis by amount of breast milk showed the lowest NEC rate in infants with high breast milk consumption and probiotics (statistically not significant).

\section{Analysis of NEC Risk Factors}

Infants developing NEC $(n=40)$ were more immature (NEC: $26+2(23+4-33+0)$ vs. no NEC: $28+0(22+6-33+6) ; P=0.005)$ than infants without NEC $(n=423)$. Premature rupture of membranes as cause for preterm birth was observed more often in infants with NEC (NEC: $62.5 \%$ vs. no NEC: $42.1 \%$; $P=0.009$ ), and these infants also received prenatal antibiotics more frequently (NEC: 52.5 vs. no NEC: $44.9 \%$; $P=0.024$ ). All other basic clinical and demographic data did not differ significantly (data not shown). Infants with NEC received significantly less breast milk during their first $2 \mathrm{wk}$ of life (NEC: $117.3 \mathrm{ml} / \mathrm{kg}$ vs. no NEC: $339.3 \mathrm{ml} / \mathrm{kg} ; P<0.001)$ but a comparable amount of formula (NEC: 186.7 vs. no NEC: $210.7 \mathrm{ml} /$ kg; $P=0.889$ ).

\section{DISCUSSION}

The probiotic Infloran (Laboratorio Farmaceutico) had no significant impact on the overall NEC rates in a prospectively followed middle European cohort of VLBW infants. In a subgroup analysis, Infloran (Laboratorio Farmaceutico) significantly reduced NEC in infants fed breast milk but was ineffective in infants exclusively fed formula. The severity of NEC and feeding tolerance were not affected.

The clinical effects of probiotics are highly specific and show large variations depending on the nature of the strain (13). Our results show that even the effect of the same probiotic 
Table 1. Demographic and basic clinical parameters

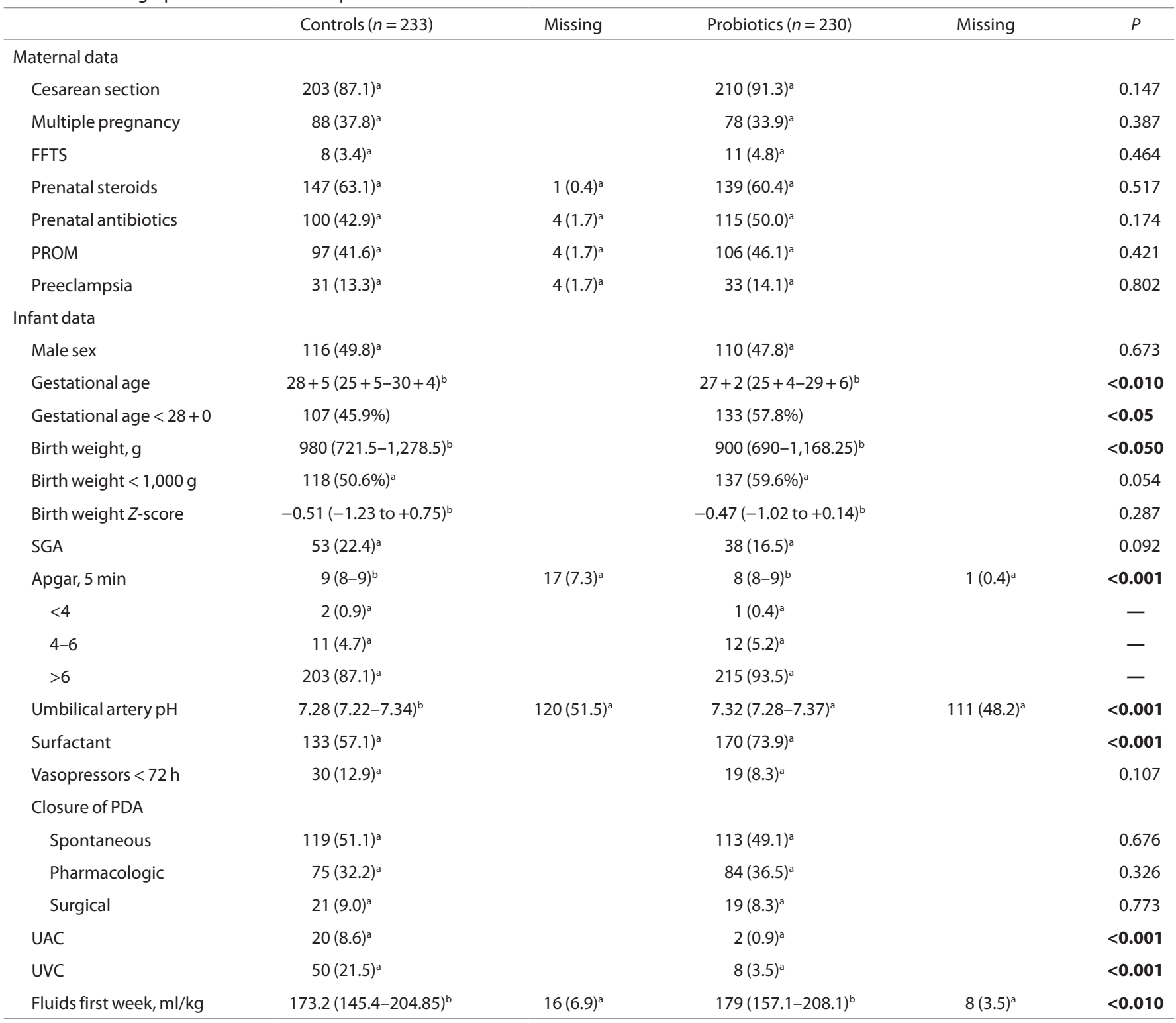

$P$ values $<0.05$ were considered statistically significant and printed in bold letters.

FFTS, feto-fetal transfusion syndrome; PDA, patent ductus arteriosus; PROM, premature rupture of membranes; SGA, small for gestational age; UAC, umbilical artery catheter; UVC, umbilical vein catheter.

aNumbers with percentage in parentheses. ${ }^{\mathrm{b}}$ Median and interquartile range.

preparation can vary considerably between different studies: Using Infloran (Laboratorio Farmaceutico), Lin et al. (15) demonstrated a sharp reduction of NEC by $80 \%$; Hoyos (16) reported on a more moderate effect (60\%), whereas we found a reduction of $32 \%$ which was not statistically significant. It seems unlikely that differences in dosage are the cause as the log units of probiotic germs were highest in our study. Moreover, the dosage did not directly correlate with the effect (here: $4 \times 10^{9}$ vs. Lin et al. (15): $2 \times 10^{9} / \mathrm{kg}$ and Hoyos (16): $\left.0.25 \times 10^{9}\right)$. Also, the smaller effect in our study may be accounted for by practice differences between different units. For this reason, it is hard to derive dosage recommendations from this and other studies. Amounts of $10^{9-10} \log$ units of probiotic germs are generally needed to overcome gastric acid, bile, and digestive enzymes in adults (17). As the digestive components in preterm infants are immature, it is thinkable that also lower doses would be sufficient or maybe even more effective, but only large multicenter trials comparing different dosing of the same probiotic could clarify this question (13).

Additionally, the weak effect of Infloran (Laboratorio Farmaceutico) in our population is not explained by a generally low NEC incidence, as the baseline incidence in the control group of our study was higher (10.3\%) compared with Lin et al. (15) (5.3\%) or Hoyos (16) (6.6\%). Also, confounding variables did not influence our results significantly, as shown by a multivariate analysis including birth weight, use of mother's milk, premature rupture of membranes, prenatal antibiotics, and death. We cannot exclude that the susceptibilities of the 
Table 2. Neonatal outcome

\begin{tabular}{|c|c|c|c|c|c|}
\hline & Controls $(n=233)$ & Missing & Probiotics $(n=230)$ & Missing & $P$ \\
\hline NEC & $24(10.3)^{\mathrm{a}}$ & & $16(7.0)^{\mathrm{a}}$ & & 0.200 \\
\hline Death & $30(12.9)^{a}$ & & $16(7.0)^{\mathrm{a}}$ & & $<0.050$ \\
\hline FIP & $1(0.4)^{\mathrm{a}}$ & & $3(1.3)^{\mathrm{a}}$ & $2(0.9)^{\mathrm{a}}$ & 0.305 \\
\hline IVH III/IV & $25(10.7)^{\mathrm{a}}$ & $1(0.4)$ & $16(7.0)^{\mathrm{a}}$ & & 0.215 \\
\hline Bronchopulmonary dysplasia & $32(13.7)^{\mathrm{a}}$ & $1(0.4)$ & $39(17.0)^{\mathrm{a}}$ & $2(0.9)^{\mathrm{a}}$ & 0.518 \\
\hline ROP requiring treatment & $21(9.0)^{\mathrm{a}}$ & $1(0.4)$ & $38(16.5)^{\mathrm{a}}$ & $6(2.6)^{\mathrm{a}}$ & $<0.010$ \\
\hline Sepsis (culture proven) & $0(0-1)^{\mathrm{b}}$ & $1(0.4)$ & $0(0-1)^{\mathrm{b}}$ & $5(2.2)^{\mathrm{a}}$ & 0.150 \\
\hline 0 & $154(66.1)^{\mathrm{a}}$ & & $165(71.7)^{\mathrm{a}}$ & & - \\
\hline 1 & $67(28.8)^{\mathrm{a}}$ & & $45(19.6)^{\mathrm{a}}$ & & - \\
\hline Sepsis with probiotic & $0(0-0)^{\mathrm{b}}$ & & $0(0-0)^{b}$ & & 1.000 \\
\hline
\end{tabular}

$P$ values $<0.05$ were considered statistically significant and printed in bold letters.

FIP, focal intestinal perforation; IVH, intraventricular hemorrhage; ROP, retinopathy of prematurity.

anumbers with percentage in parentheses. ${ }^{\mathrm{b} M e d i a n}$ and interquartile range.

Table 3. Multivariate analysis

\begin{tabular}{lcccccc}
\hline & $B$ & SE & Wald & df & $P$ & $\operatorname{Exp}(B)$ \\
\hline Probiotics & -0.440 & 0.361 & 1.481 & 1 & 0.224 & 0.644 \\
Birth weight & -0.001 & 0.001 & 2.740 & 1 & 0.098 & 0.999 \\
Any BM & 0.096 & 0.437 & 0.048 & 1 & 0.827 & 1.100 \\
PROM & 0.496 & 0.389 & 1.624 & 1 & 0.202 & 1.642 \\
Prenatal antibiotics & 0.574 & 0.394 & 2.123 & 1 & 0.145 & 1.776 \\
Death & 1.255 & 0.455 & 7.612 & 1 & $<\mathbf{0 . 0 5 0}$ & 3.509 \\
Constant & -1.554 & 0.995 & 2.436 & 1 & 0.119 & 0.211 \\
\hline
\end{tabular}

Binary logistic regression analysis on the effect of probiotics, controlled for the

confounding covariates birth weight, any breast milk in the first 2 wk of life, PROM,

prenatal antibiotics, and death. $P$ values $<0.05$ were considered statistically significant and printed in bold letters.

BM, breast milk; PROM, premature rupture of membranes.

study populations were different. The study by Lin et al. (15) was conducted in China, Hoyos (16) reported data from South America, and the population cared for at our hospital is mainly Caucasian. Similar results concerning the efficacy of probiotics in different ethnicities were also found in two studies that investigated the probiotic ABCDophilus (a mixture of B. infantis, Bifidobacterium bifidum, and Streptococcus thermophilus; Solgar, Leonia, NJ). While Bin-Nun et al. (18) from Israel demonstrated a reduction of NEC by $90 \%$ (from 10 to $1 \%$ ), the ProPrems trial (19) in Australia and New Zealand found a more modest reduction by 55\% (from 4.4 to $2 \%$ ). Variations in susceptibility to certain drugs among different ethnicities were previously described (20) and could also be relevant with probiotics. In this respect, it is well established that probiotics interact with the host's immune system via toll-like receptors (21) that display polymorphisms between different ethnicities
(22), which may modulate the preventive effect of a probiotic against NEC. Currently, there is no study or meta-analysis that addresses the question whether the effect of a probiotic is influenced by race, and systematic investigations could be of interest.

Breast milk is a well-established option for preventing NEC (23), and accordingly, NEC was associated with less intake of breast milk during the first 2 wk of life in our study. Yet, what remained unclear so far is how the source of enteral nutrition (breast milk or formula) influences on the efficacy of probiotics in preventing NEC (13). One would expect that infants fed formula would especially benefit from probiotics, as these infants present with a reduced colonization with bifidobacteria and lactobacilli (24). To our surprise, the preventive effect of Infloran (Laboratorio Farmaceutico) against NEC was restricted to infants who received any breast milk, providing evidence that the efficacy of the probiotic germs depends on concomitant feeding of human milk. Oligosaccharides in breast milk contribute to the growth of bifidobacteria and lactobacilli (25), which may enhance the effect of these germs preventing NEC also after oral supplementation. In this regard, the lowest NEC rate was found in infants who received probiotics and were fed high amounts of breast milk. Although analyses of subgroups need to be interpreted with caution, it seems unlikely from our data that infants exclusively fed formula had any benefit from Infloran (Laboratorio Farmaceutico). Actually, there are no published data showing an effect of Infloran (Laboratorio Farmaceutico) or any other probiotic in infants who were solely formula fed. In this line, it is of high interest that in the study of Lin et al. (15) - where Infloran (Laboratorio Farmaceutico) was highly efficaciousinfants were exclusively fed breast milk. The fact that all infants 
Table 4. Severity of NEC

\begin{tabular}{|c|c|c|c|c|}
\hline & Controls $(n=24)$ & Missing & Probiotics $(n=17)^{\mathrm{c}}$ & $P$ \\
\hline Mortality & $7(29.2)^{\mathrm{a}}$ & & $5(31.3)^{\mathrm{a}}$ & 0.986 \\
\hline Onset of NEC (days) & $16.5(13-29)^{\mathrm{b}}$ & & $14(8.5-22)^{b}$ & 0.314 \\
\hline Highest IL-8 in $72 \mathrm{~h}$ after onset (pg/ml) & $2,135(626-4,642)^{b}$ & $2(8.3)^{a}$ & $1,236(349-7,750)^{\mathrm{b}}$ & 0.588 \\
\hline Abdominal distension & $23(95.8)^{\mathrm{a}}$ & & $17(100)^{\mathrm{a}}$ & 0.394 \\
\hline Mechanical ventilation & $19(79.2)^{\mathrm{a}}$ & $1(4.2)^{\mathrm{a}}$ & $14(82.4)^{\mathrm{a}}$ & 0.983 \\
\hline Use of vasopressors & $10(41.7)^{\mathrm{a}}$ & $1(4.2)^{\mathrm{a}}$ & $9(52.9)^{a}$ & 0.554 \\
\hline Pneumatosis & $13(54.2)^{\mathrm{a}}$ & & $6(37.5)^{a}$ & 0.184 \\
\hline Portal venous gas & $2(8.3)^{\mathrm{a}}$ & & $1(5.9)^{\mathrm{a}}$ & 0.738 \\
\hline Surgery & $14(58.3)^{\mathrm{a}}$ & & $13(76.5)^{\mathrm{a}}$ & 0.228 \\
\hline
\end{tabular}

$P$ values $<0.05$ were considered statistically significant and printed in bold letters.

IL, interleukin; NEC, necrotizing enterocolitis.

${ }^{a}$ Numbers with percentage in parentheses. ${ }^{b}$ Median and interquartile range. ${ }^{\mathrm{c}} \mathrm{No}$ missing data.

Table 5. Nutritional variables

\begin{tabular}{|c|c|c|c|c|c|}
\hline & Controls $(n=233)$ & Missing & Probiotics $(n=230)$ & Missing & $P$ \\
\hline Full enteral feeds (DOL) & $24(14-38)^{b}$ & $69(29.6)^{a}$ & $23(17-40)^{b}$ & $29(12.6)^{a}$ & 0.133 \\
\hline \multicolumn{6}{|l|}{ Analysis in the first $2 \mathrm{wk}$ of life } \\
\hline Amount of BM (ml/kg) & $265.8(60.7-576.3)^{\mathrm{b}}$ & $38(16.3)^{a}$ & $350.6(60.6-625.8)^{b}$ & $15(6.5)^{\mathrm{a}}$ & 0.384 \\
\hline Total enteral feeds $(\mathrm{ml} / \mathrm{kg})$ & $683.95(386.5-998.8)^{\mathrm{b}}$ & $38(16.3)^{\mathrm{a}}$ & $677.4(442.3-948.5)^{\mathrm{b}}$ & $15(6.5)^{\mathrm{a}}$ & 0.919 \\
\hline Bilious gastric residuals & $159(68.2)^{\mathrm{a}}$ & $6(2.6)^{\mathrm{a}}$ & $126(54.8)^{\mathrm{a}}$ & $31(13.5)^{\mathrm{a}}$ & 0.141 \\
\hline
\end{tabular}

$\mathrm{BM}$, breast milk; DOL, day of life.

anumbers with percentage in parentheses. ${ }^{\mathrm{b}}$ Median and interquartile range.

Table 6. Subgroup analysis of NEC according to feeding type

\begin{tabular}{lccc}
\hline & Controls $(n=233)$ & Probiotics $(n=227)^{\mathrm{a}}$ & $P$ \\
\hline Formula & $4 / 54(7.4)$ & $6 / 44(13.6)$ & 0.283 \\
Any BM & $20 / 179(11.2)$ & $10 / 183(5.5)$ & $<\mathbf{0 . 0 5 0}$ \\
All & $24 / 233(10.3)$ & $16 / 227(7.0)$ & 0.224 \\
Quantitative analysis & $(n=220)^{\mathrm{b}}$ & $(n=216)^{\mathrm{c}}$ & \\
$\quad$ 0-32\% BM & $12 / 97(12.3)$ & $10 / 66(15.2)$ & 0.732 \\
$33-65 \%$ BM & $6 / 46(13.0)$ & $2 / 31(6.5)$ & 0.317 \\
$>65 \%$ BM & $5 / 77(6.4)$. & $4 / 119(3.3)$ & 0.208 \\
\hline
\end{tabular}

Infants who received exclusively formula were compared with those who received any BM in the first 2 wk of life (upper table). In a quantitative analysis, groups were split up by percentage of BM as indicated (lower table). Data are presented as cases/total infants with percentages in parentheses. A binary logistic regression analysis was performed; the detailed statistic analyses of the groups"formula" and "any BM"are shown in Table 7. $P$ values $<0.05$ were considered statistically significant and printed in bold letters.

$\mathrm{BM}$, breast milk; NEC, necrotizing enterocolitis.

${ }^{a}$ Data of three patients missing. ${ }^{b}$ Data of 13 patients missing. ${ }^{\circ}$ Data of 14 patients missing.

in our study were started on formula on the first day of life and never exclusively fed breast milk might explain the weak effect of Infloran (Laboratorio Farmaceutico) in our study. In particular, early exposure to formula may have an impact on gut colonization patterns and NEC risk. From our findings and the published literature, it is therefore reasonable to conclude that avoidance of early exposure to formula will help to improve the efficacy of probiotics against NEC. Further studies on probiotics and their benefit under different types of enteral feeding are urgently needed to confirm our findings. Further trials should also consider a potentially lower effect of probiotics in infants exclusively fed formula regarding power calculations.

Some studies suggest that probiotics also reduce the severity of NEC. Using Infloran (Laboratorio Farmaceutico), Lin et al. (15) showed that 6/10 NEC cases in the control group, but only $0 / 2$ in the study group, were Bell stage 3. Bin-Nun et al. (18) also reported a significant reduction in NEC severity using ABCDophilus (Solgar). In our study, a detailed analysis of NEC cases including clinical and laboratory parameters did not reveal any changes in mortality, severe NEC (Bell's stage IIIb), or the clinical course of NEC. Platelet levels were even significantly lower in NEC cases of the probiotics group, which may rather indicate a greater disease extent (26). Therefore, our 
data do not support that Infloran (Laboratorio Farmaceutico) reduces NEC severity.

Death was significantly lower in infants who received probiotics in our study, but the positive effect on survival even exceeded the reduction of NEC, which is not seen in the meta-analyses $(13,14)$. Consequently, it can be assumed that other factors besides a reduction of NEC improved the survival in these infants. NEC, severe IVH, and sepsis are major contributors to death among VLBW infants (27). The reduction of mortality in our study-as it can be explained from our data-is caused by the reduction of NEC and severe IVH that was statistically significant as a compound outcome-as well as improvements in sepsis management. Besides probiotics, we introduced pentoxifylline to reduce mortality in severe sepsis. In accordance with the protective effect of pentoxifylline against death in sepsis (28), cases of lethal sepsis have halved, while the incidence of sepsis did not change markedly. It is however a limitation that we do not have the data on the use of pentoxifylline in all study infants. Furthermore, a way of less invasive surfactant application was established that improved the survival of infants $<26 \mathrm{wk}$ GA and reduced severe IVH (29) and was accompanied by improvements in physician's staffing which itself has a known impact on patient's mortality (30). Overall, we conclude that reduced morbidities and improvements in the management of VLBW infants-not probiotics alone-have significantly reduced mortality in our cohort. In this respect, we also observed a trend toward a lower mortality during the first week of life when probiotics are rather unlikely to be effective and NEC is uncommon (infants that were not eligible due to death in the first week of life: $37 / 340$ (10.9\%) vs. 22/326 (6.7\%); $P=0.086$; Figure 1).

Table 7. Multivariate analysis on the effect of probiotics in infants according to feeding type

\begin{tabular}{lcccccc}
\hline Formula & $B$ & SE & Wald & df & $P$ & Exp $(B)$ \\
\hline Probiotics & 0.940 & 0.875 & 1.153 & 1 & 0.283 & 2.559 \\
Birth weight & -0.003 & 0.002 & 2.914 & 1 & 0.088 & 0.997 \\
PROM & -0.540 & 0.990 & 0.298 & 1 & 0.585 & 0.583 \\
Prenatal antibiotics & 1.856 & 0.987 & 3.535 & 1 & 0.060 & 6.396 \\
Death & 0.943 & 0.948 & 0.990 & 1 & 0.320 & 2.567 \\
Constant & -1.945 & 2.342 & 0.690 & 1 & 0.406 & 0.143 \\
Any breast milk & & & & & & \\
Probiotics & -0.844 & 0.421 & 4.027 & 1 & $<\mathbf{0 . 0 5 0}$ & 0.430 \\
Birth weight & -0.001 & 0.001 & 0.987 & 1 & 0.321 & 0.999 \\
PROM & 0.677 & 0.442 & 2.343 & 1 & 0.126 & 1.968 \\
Prenatal antibiotics & 0.224 & 0.441 & 0.258 & 1 & 0.612 & 1.251 \\
Death & 1.510 & 0.521 & 8.404 & 1 & $<\mathbf{0 . 0 5 0}$ & 4.527 \\
Constant & -1.167 & 1.022 & 1.305 & 1 & 0.253 & 0.311
\end{tabular}

Binary logistic regression analysis on the effect of probiotics in infants fed exclusively formula (upper table) or any breast milk (lower table), controlled for the confounding covariates birth weight, premature rupture of membranes, prenatal antibiotics, and death. $P$ values $<0.05$ were considered statistically significant and printed in bold letters.

PROM, premature rupture of membranes.
The significant increase of retinopathy of prematurity in the probiotics group seen in the univariate analysis was investigated in a model of logistic regression correcting for GA and death showing that it was not attributable to probiotics but to the improved survival rate and lower GA.

In a recent trial, a significant reduction of culture-proven sepsis (but not NEC) was shown using Lactobacillus reuteri (31). We did not find any influence on culture-proven sepsis in our study using Infloran (Laboratorio Farmaceutico), which is in line with other investigations $(19,32)$. As in other studies (33), sepsis with probiotic germs did not occur-neither in the study population nor in infants excluded from the study (data not shown)-which is reassuring.

There are some reports on a positive influence of probiotics on feeding tolerance. Kitajima et al. (34) found less gastric aspirates (Bifidobacterium breve), while Rougé et al. (35) (mixture of Lactobacillus rhamnosus GG and Bifidobacterium longum) and Oncel et al. (31) (L. reuteri) demonstrated reductions in time to full enteral feeds. We did not find any improvements in this regard.

Limitations of our study are the retrospective nature of controls, the more as concomitant changes in practice of airway and sepsis management occurred in the study period and survival generally improved. However, prophylactic surfactant and pentoxifylline have no published effect on NEC but may have influenced some of the secondary outcomes.

In summary, our study showed that the efficacy of Infloran (Laboratorio Farmaceutico) varies strongly between centers and was of low efficacy in our population of VLBW infants. A preventive effect against NEC was only present in infants fed breast milk in the first $2 \mathrm{wk}$ of life-but not in infants exclusively fed formula-suggesting that the efficacy of probiotics is strongly influenced by feeding practices. We found no influence on NEC severity or improvement in feeding tolerance. Future studies should urgently focus on the use of probiotics in infants fed formula compared with breast milk.

\section{METHODS}

\section{Probiotics and Enteral Feedings}

From September 2010, all preterm infants $<34+0$ wk GA admitted to our unit received Infloran (Laboratorio Farmaceutico, Sanremo, Italy; one capsule containing $10^{9} \mathrm{~L}$. acidophilus and $10^{9} \mathrm{~B}$. infantis; storage at $4{ }^{\circ} \mathrm{C}$ ) from birth until discharge or $37+0 \mathrm{wk}$ GA. The drug was applied twice a day with enteral feedings. Infloran (Laboratorio Farmaceutico) was not used in infants with gut malformations and discontinued if definite NEC occurred. In all infants, enteral feedings were started on the first day of life using preterm formula $(1 \mathrm{ml}$ every $3 \mathrm{~h}$ ) and advanced by $10-20 \mathrm{ml} / \mathrm{kg} / \mathrm{d}$ depending on feeding tolerance. Feedings were switched to breast milk as soon as it was available from the infant's mother. If no breast milk was available, infants remained on preterm formula. Fortification of breast milk was started as soon as $100 \mathrm{ml} / \mathrm{kg} / \mathrm{d}$ of enteral feedings were reached, using a bovine milk fortifier.

\section{Study Design and Eligibility}

We performed an observational study on the effects of Infloran (Laboratorio Farmaceutico) in VLBW $(<1,500 \mathrm{~g})$ infants born $<34 \mathrm{wk}$ of gestation. Infants in the prospective study group received Infloran (Laboratorio Farmaceutico) ("probiotics" October 2010-July 2012) and were compared with a retrospective control group receiving no probiotics ("controls" January 2008-December 2009). Patients were 
excluded from the study due to the following conditions: (i) patients who died in the first week of life (except due to NEC), (ii) patients who did not receive Infloran (Laboratorio Farmaceutico) from the first week of life to $34 \mathrm{wk}$ GA (except discontinuation after NEC), (iii) patients who were transferred to another hospital before $34 \mathrm{wk}$ GA (except for a partner clinic (Wilhelminen Hospital Vienna) that implemented the same probiotic regime and provided data of transferred infants) and (iv) patients with gut malformations. The primary objective was to determine the effect of probiotics on NEC incidence compared with a historic control group. Secondary objectives were to evaluate the impact of probiotics on NEC severity and to analyze whether the type of enteral feeding in the first $2 \mathrm{wk}$ of life (any breast milk vs. exclusively formula) acts upon the effect of probiotics on NEC incidence. Other objectives were to evaluate safety concerning culture-proven sepsis in context with probiotics intake and the influence on feeding tolerance.

\section{Data Collection}

Data were retrieved from the electronic patient's charts (ICCA, Phillips Medical Systems, Eindhoven, The Netherlands), the medical picture archiving and communication system (IMPAX, Agfa HealthCare, Bonn, Germany), and discharge letters.

Demographic and basic clinical parameters. Small for gestational age was defined as birth weight $<10$ th percentile and prenatal steroids as a full course (two doses) of betamethasone (Table 1). Closure of patent ductus arteriosus was diagnosed by cardiac ultrasound. Surfactant $(200 \mathrm{mg} / \mathrm{kg}$ Curosurf; Chiesi, Parma, Italia) was used therapeutically in preterm infants with more than $35 \%$ oxygen on continuous positive airway pressure in the first $2 \mathrm{~h}$ after birth. Additionally-starting from 2009-infants $<28 \mathrm{wk}$ GA received surfactant prophylactically using less invasive surfactant application (29). Vasopressors in the first $3 \mathrm{~d}$ of life were used if the mean arterial blood pressure was below the gestational week at birth. Pentoxifylline $(30 \mathrm{mg} / \mathrm{kg}$ for $5 \mathrm{~d})(28)$ use in infants with septicemia and NEC was started from 2009 if infants showed failure of one or more organ systems or high inflammatory parameters (i.e., interleukin-8 $>1,000$ ). Infants did not receive lactoferrin. Oxygen saturation target ranges were $83-95 \%$ for $<32$ wk GA and $85-96 \%$ for $\geq 32$ wk GA.

Neonatal outcome. The primary outcome parameter was definite NEC diagnosed clinically (modified Bell's stage $\geq$ IIa (36)) with pneumatosis as mandatory radiological criterion (Tables 2 and 3 ). Surgical diagnosis was made in those cases where pneumatosis was not present on $\mathrm{x}$-ray, but direct visualization revealed inflammatory bowel without other pathology (e.g., volvulus). Focal intestinal perforation was diagnosed if a single intestinal perforation occurred in an otherwise healthy bowel. Other neonatal outcome parameters were: death before discharge, IVH grade III/IV and cystic periventricular leucomalacia (diagnosed by ultrasound $(37,38)$ performed every 1-2wk), bronchopulmonary dysplasia (supplementary oxygen after $36 / 0 \mathrm{wk}$ GA), retinopathy of prematurity requiring treatment (laser or antivascular endothelial growth factor), culture-proven septicemia (blood cultures drawn by sterile phlebotomy of $0.5 \mathrm{ml}$ blood using BacT/Alert Pediatric FAN blood culture media (BioMerieux, Marcy l'Etoile, France)), lethal sepsis, and sepsis with a probiotic germ. The culturability of the two probiotic strains using BacT/Alert Pediatric FAN (Bio Merieux) was confirmed by the hospital's microbiology department (data not shown).

Analysis of NEC severity. NEC cases in the control and probiotics groups were compared for mortality, perforation (i.e., NEC stage IIIb), laboratory parameters (thrombocyte nadir the next $7 \mathrm{~d}$ (39) and interleukin-8 peak (40) within $72 \mathrm{~h}$ after NEC onset), descriptive clinical parameters (NEC onset, abdominal distension, bloody stools, mandatory ventilation, use of vasopressors, and surgery), and radiological signs (pneumatosis and portal venous gas; Table 4).

Nutritional variables. Data on feeding type were collected during the first 2 wk of life (associated with protection from NEC according to Sisk et al. (5)), and the total amount of breast milk vs. formula was recorded (expressed as $\mathrm{ml} / \mathrm{kg}$ body weight; Table 5). Feeding tolerance in the first $2 \mathrm{wk}$ of life was determined by analyzing the amount of gastric residuals and presence of bilious residuals. Days until full enteral feeds were defined as reaching $140 \mathrm{ml} / \mathrm{kg}$ enteral intake or discontinuation of parenteral nutrition-whatever occurred earlier.

Subgroup analysis according to feeding type. The two study groups were separated according to their type of enteral nutrition in the first 2 wk of life (exclusively formula vs. any breast milk; Tables 6 and 7). Furthermore, infants were analyzed by amount of breast milk (no/low: 0-32\%, intermediate: $33-65 \%$, and high percentage of breast milk: $>65 \%$ ). The NEC incidence in the control and probiotics groups was compared as described in the Statistics section.

Analysis of NEC risk factors. Differences in demographic and basic clinical data as well as nutritional variables were evaluated.

\section{Statistics}

We anticipated a reduction of NEC by $65 \%$, as published in the metaanalyses $(13,14)$ and calculated a sample size of 226 patients per group $(\alpha$-error $<0.05$; power $80 \%)$, based on a baseline NEC incidence of $10.3 \%$. Statistical analysis was performed using the $\chi^{2}$ test for categorical data; Student's $t$-test and Mann-Whitney $U$-test were used for continuous variables. A multivariate analysis was performed for the primary outcome (including subgroup analyses) using a model of binary logistic regression that included the confounding covariates birth weight, any mothers' milk in the first $2 \mathrm{wk}$ of life (not for subgroup analyses), premature rupture of membranes, prenatal antibiotics, and death. The impact of probiotics on retinopathy of prematurity (significantly higher in the univariate analysis) was controlled for the influence of GA and mortality in a model of binary logistic regression as well. $P$ values $<0.05$ were regarded as statistically significant.

\section{Ethics and Registration}

As probiotics were introduced for routine therapy and controls were analyzed retrospectively, informed consent was not considered necessary by the institute's ethics board. The ethics committee of the Medical University of Vienna approved the study design. The study was registered at clinicaltrials.gov (NCT01751477).

\section{STATEMENT OF FINANCIAL SUPPORT}

No financial assistance was received to support this study.

Disclosures: The authors state that there are no financial ties to products used in the study or potential/perceived conflicts of interest.

\section{REFERENCES}

1. Schnabl KL, Van Aerde JE, Thomson AB, Clandinin MT. Necrotizing enterocolitis: a multifactorial disease with no cure. World J Gastroenterol 2008; 14:2142-61.

2. Lin PW, Stoll BJ. Necrotising enterocolitis. Lancet 2006;368:1271-83.

3. Berseth CL. Feeding strategies and necrotizing enterocolitis. Curr Opin Pediatr 2005;17:170-3.

4. McCallie KR, Lee HC, Mayer O, Cohen RS, Hintz SR, Rhine WD. Improved outcomes with a standardized feeding protocol for very low birth weight infants. J Perinatol 2011;31:Suppl 1:S61-7.

5. Sisk PM, Lovelady CA, Dillard RG, Gruber KJ, O'Shea TM. Early human milk feeding is associated with a lower risk of necrotizing enterocolitis in very low birth weight infants. J Perinatol 2007;27:428-33.

6. Kligler B, Cohrssen A. Probiotics. Am Fam Physician 2008;78:1073-8.

7. Borriello SP, Hammes WP, Holzapfel W, et al. Safety of probiotics that contain lactobacilli or bifidobacteria. Clin Infect Dis 2003;36:775-80.

8. Ohland CL, Macnaughton WK. Probiotic bacteria and intestinal epithelial barrier function. Am J Physiol Gastrointest Liver Physiol 2010;298:G80719.

9. Williams NT. Probiotics. Am J Health Syst Pharm 2010;67:449-58.

10. Pagnini C, Saeed R, Bamias G, Arseneau KO, Pizarro TT, Cominelli F. Probiotics promote gut health through stimulation of epithelial innate immunity. Proc Natl Acad Sci USA 2010;107:454-9.

11. Corthésy B, Gaskins HR, Mercenier A. Cross-talk between probiotic bacteria and the host immune system. J Nutr 2007;137:Suppl 2:781S-90S.

12. Claud EC, Walker WA. Hypothesis: inappropriate colonization of the premature intestine can cause neonatal necrotizing enterocolitis. FASEB J 2001;15:1398-403. 
13. Deshpande G, Rao S, Patole S, Bulsara M. Updated meta-analysis of probiotics for preventing necrotizing enterocolitis in preterm neonates. Pediatrics 2010;125:921-30.

14. Guthmann F, Kluthe C, Bührer C. Probiotics for prevention of necrotising enterocolitis: an updated meta-analysis. Klin Padiatr 2010;222:284-90.

15. Lin $\mathrm{HC}, \mathrm{Su} \mathrm{BH}$, Chen $\mathrm{AC}$, et al. Oral probiotics reduce the incidence and severity of necrotizing enterocolitis in very low birth weight infants. Pediatrics 2005;115:1-4.

16. Hoyos AB. Reduced incidence of necrotizing enterocolitis associated with enteral administration of Lactobacillus acidophilus and Bifidobacterium infantis to neonates in an intensive care unit. Int J Infect Dis 1999;3:197-202.

17. Farnworth ER. The evidence to support health claims for probiotics. J Nutr 2008; 138:1250S-4S.

18. Bin-Nun A, Bromiker R, Wilschanski M, et al. Oral probiotics prevent necrotizing enterocolitis in very low birth weight neonates. J Pediatr 2005;147:192-6.

19. Garland SM, Tobin JM, Pirotta M, et al.; ProPrems Study Group. The ProPrems trial: investigating the effects of probiotics on late onset sepsis in very preterm infants. BMC Infect Dis 2011;11:210.

20. Ortega VE, Meyers DA. Pharmacogenetics: implications of race and ethnicity on defining genetic profiles for personalized medicine. J Allergy Clin Immunol 2014;133:16-26.

21. Bermudez-Brito M, Plaza-Díaz J, Muñoz-Quezada S, Gómez-Llorente C, Gil A. Probiotic mechanisms of action. Ann Nutr Metab 2012;61:160-74.

22. Netea MG, Wijmenga C, O'Neill LA. Genetic variation in Toll-like receptors and disease susceptibility. Nat Immunol 2012;13:535-42.

23. Meinzen-Derr J, Poindexter B, Wrage L, Morrow AL, Stoll B, Donovan EF. Role of human milk in extremely low birth weight infants' risk of necrotizing enterocolitis or death. J Perinatol 2009;29:57-62.

24. Carlisle EM, Morowitz MJ. The intestinal microbiome and necrotizing enterocolitis. Curr Opin Pediatr 2013;25:382-7.

25. Zivkovic AM, German JB, Lebrilla CB, Mills DA. Human milk glycobiome and its impact on the infant gastrointestinal microbiota. Proc Natl Acad Sci USA 2011;108:Suppl 1:4653-8.

26. Ververidis M, Kiely EM, Spitz L, Drake DP, Eaton S, Pierro A. The clinical significance of thrombocytopenia in neonates with necrotizing enterocolitis. J Pediatr Surg 2001;36:799-803.

27. Stichtenoth G, Demmert M, Bohnhorst B, et al. Major contributors to hospital mortality in very-low-birth-weight infants: data of the birth year 2010 cohort of the German Neonatal Network. Klin Padiatr 2012;224:276-81.
28. Haque KN, Pammi M. Pentoxifylline for treatment of sepsis and necrotizing enterocolitis in neonates. Cochrane Database Syst Rev 2011;CD004205.

29. Klebermass-Schrehof K, Wald M, Schwindt J, et al. Less invasive surfactant administration in extremely preterm infants: impact on mortality and morbidity. Neonatology 2013;103:252-8.

30. Pronovost PJ, Angus DC, Dorman T, Robinson KA, Dremsizov TT, Young TL. Physician staffing patterns and clinical outcomes in critically ill patients: a systematic review. JAMA 2002;288:2151-62.

31. Oncel MY, Sari FN, Arayici S, et al. Lactobacillus reuteri for the prevention of necrotising enterocolitis in very low birthweight infants: a randomised controlled trial. Arch Dis Child Fetal Neonatal Ed 2014;99: F110-5.

32. Mihatsch WA, Vossbeck S, Eikmanns B, Hoegel J, Pohlandt F. Effect of Bifidobacterium lactis on the incidence of nosocomial infections in verylow-birth-weight infants: a randomized controlled trial. Neonatology 2010;98:156-63.

33. Ofek Shlomai N, Deshpande G, Rao S, Patole S. Probiotics for preterm neonates: what will it take to change clinical practice? Neonatology 2014;105:64-70.

34. Kitajima H, Sumida Y, Tanaka R, Yuki N, Takayama H, Fujimura M. Early administration of Bifidobacterium breve to preterm infants: randomised controlled trial. Arch Dis Child Fetal Neonatal Ed 1997;76:F101-7.

35. Rougé $\mathrm{C}$, Piloquet $\mathrm{H}$, Butel MJ, et al. Oral supplementation with probiotics in very-low-birth-weight preterm infants: a randomized, double-blind, placebo-controlled trial. Am J Clin Nutr 2009;89:1828-35.

36. Gregory KE, Deforge CE, Natale KM, Phillips M, Van Marter LJ. Necrotizing enterocolitis in the premature infant: neonatal nursing assessment, disease pathogenesis, and clinical presentation. Adv Neonatal Care 2011;11:155-64; quiz 165-6.

37. de Vries LS, Eken P, Dubowitz LM. The spectrum of leukomalacia using cranial ultrasound. Behav Brain Res 1992;49:1-6.

38. Volpe JJ. Intraventricular hemorrhage and brain injury in the premature infant. Diagnosis, prognosis, and prevention. Clin Perinatol 1989;16:387411.

39. Kling PJ, Hutter JJ. Hematologic abnormalities in severe neonatal necrotizing enterocolitis: 25 years later. J Perinatol 2003;23:523-30.

40. Benkoe T, Reck C, Gleiss A, et al. Interleukin 8 correlates with intestinal involvement in surgically treated infants with necrotizing enterocolitis. J Pediatr Surg 2012;47:1548-54. 